\title{
The tradition of lyric poetry in the Chhayavadi era
}

\author{
Rakhi Upadhyay* \\ Department of Hindi DAV PG College Dehradun \\ *Corresponding Author: drrakhi_418@rediffmail.com \\ Received: 8.6.2021; Revised: 19.12.2021; Accepted: 29.12.2021 \\ (O) Society for Himalayan Action Research and Development
}

\begin{abstract}
:
The song has been considered complete. It was acknowledged that the song's greatest quality was that it was complete. In the modern era, the word song has taken many forms, both literally and economically. The word "Geet" has not only evolved in terms of meaning and impact, but it has evolved equally from the morphological point of view. Words like Geeti Geet Kavya or Prageeti Kavi, Prageeti Muktak evolved from the song itself. The words Geet and Geeti have been used since ancient times. Poetry in its infancy was a song. From the beginning till today, the importance of this song or geet poetry is kept in its summing up. Generally, the method of giving lyrical expression to the poet's personal sentiment and feeling is called Gita Kavya.
\end{abstract}

Key words: Poetry, Meaning, Uses, Chhayavadi Lyrics.

\section{छायावादीयुग में गीतिकाव्य की परम्परा}

\section{राखी उपाध्याय}

हिन्दी विभाग,

डी.ए.वी. (पी.जी.) कॉलेज, देहरादून

ई-मेल drrakhi_418@rediffmail.com

\section{सारांश}

गीत को अपने आप में परिपूर्ण माना जाता रहा है। यह स्वीकार किया गया कि "गीत की सबसे बड़ी खूबी है कि वह अपने आप में परिपूर्ण है। "गीत वह है जिसमें भाव विचार अनुभूति कल्पना- एक शब्द में कथ्य की एकता है। आधुनिक युग में इस गीत शब्द ने शाब्दिक तथा आर्थिक दोनों ही प्रकार से अनेक रूप धारण किया है- "गीत" शब्द का न केवल अर्थ और प्रभाव की दृष्टि से विकास हुआ है वरन् रूपात्मक दृष्टि से भी वह उतना ही विकसित हुआ है गीत से गीति-गीत काव्य अथवा गीति काव्य, प्रगीति अथवा प्रगीति काव्य, प्रगीत मुक्तक जैसे शब्दों का निर्माण हुआ है। गीत तथा गीति शब्द दोनों का ही प्रयोग नया नहीं है- इसका प्रयोग अत्यन्त प्राचीन काल से होता आ रहा है। प्राचीन शास्त्रीय चिन्तन के बाद हिन्दी में 
काव्य रूपों को लेकर आधुनिक युग से पहले गम्भीर चिन्तन बहुत कम हुआ है। छायावादी कवियों ने गीत को व्यक्तिगत सीमा में ही तीव्र सुख-दुखात्मक अनुभूति का शब्द रूप स्वीकार किया, जो अपनी ध्वन्यात्मकता में गेय हो सकता था। उन्होंने सुख-दुख की भावावेशमयी अवस्था विशेष का गिने-चुने शब्दों में स्वर साधना के द्वारा उपयुक्त चित्रण कर देने को 'गीत' की संज्ञा दी। "काव्य अपनी आरम्भिक अवस्था में गीत ही था। आरम्भ से लेकर आज तक इस गीत अथवा गीति काव्य की महत्ता उसके संक्षेपीकरण में रखी है। सामान्यतः कवि की वैयक्तिक भाव धारा और अनुभूति को उसके अनुरूप लयात्मक अभिव्यक्ति देने के विधान को गीति काव्य कहते हैं।

कुँजी शब्द-गीत शब्द का अर्थ तथा प्रयोग, छायावादी काव्य

आधुनिक युग में छायावादी कवियों ने अपनी रचनाओं के प्रति द्विवेदी युगीन आचार्यों का जो मत था वह अच्छा नहीं था और उनके विषय में अनेकानेक भ्रम भी प्रचलित किए जा रहे थे। इसके साथ ही आधुनिक युग प्राचीनता से बहुत आगे बढ़ चुका था।

छायावादी कवियों ने गीत को व्यक्तिगत सीमा में ही तीव्र सुख-दुखात्मक अनुभूति का शब्द रूप स्वीकार किया, जो अपनी ध्वन्यात्मकता में गेय हो सकता था। उन्होंने सुख-दुख की भावावेशमयी अवस्था विशेष का गिने-चुने शब्दों में स्वर साधना के द्वारा उपयुक्त चित्रण कर देने को 'गीत' की संज्ञा दी। वैशिष्ट्य के रूप में यह स्वीकार किया गया कि इसका महात्मय गायक के आलाप के साथ ही स्पष्ट हो जाता है। महादेवी वर्मा ने गीतों की रचना के साथ ही इस पर विचार भी प्रस्तुत किया। समालोचना संबंधी निबन्धों के माध्यम से उन्होंने कुल मिला जिस बात पर बल दिया वह भाव की तीव्रता की थी। यह भाव तीव्रता भी दूसरों के उल्लास विलास के साथ तादात्म्य स्थापित करने से ही प्राप्त हो सकती है- ऐसा माना गया।

कालान्तर में 'गीतों' की अन्विति को विशेष महत्त्व दिया गया। कहा गया है कि "गीतों की अपनी एक इकाई होती है। भावों विचारों की ओर एक हद तक अभिव्यक्ति के उपकरणों की भी- उनका आनन्द लेने के लिए किसी टीका टिप्पणी की आवश्यकता नहीं होती।"1

गीत को अपने आप में परिपूर्ण माना जाता रहा है। यह स्वीकार किया गया कि "गीत की सबसे बड़ी खूबी है कि वह अपने आप में परिपूर्ण है। इसके लिए सन्दर्भ या प्रसंग की आवश्यकता नहीं है।"2

इस प्रकार गीत में भाव विचार, अनुभूति और कल्पना को एक साथ इस रूप में अभिव्यक्त करने में बात कही गई है कि उसका प्रभाव भी एक साथ वैसा ही पड़ सके। "गीत वह है जिसमें भाव विचार अनुभूति कल्पना- एक शब्द में कथ्य की एकता है। और उसका एक ही प्रभाव पड़े।"3 गीत शब्द को परिभाषित करने से पूर्व कुछ प्रमुख तत्त्वों को ध्यान में रखा गया- उदाहरणार्थ "गीत" शब्द का प्रयोग ऐसी रचनाओं के लिए हुआ जिनमें अनुभूति के धरातल पर अभिव्यक्ति 
का स्वर इस प्रकार अनुभूत हुआ कि- अभिव्यंजना के उस क्षण विशेष में कवि स्वयं केन्द्र में विद्यमान था। कवि जो कुछ कहना चाह रहा था वह सब अपने पर बीता हुआ यथार्थ था। उसमें उसके 'स्व' की पुकार थी। उसमें गेयात्मकता थी, लयात्मकता और रागात्मकता स्वाभाविक रूप से प्रकट हुई थी। यह अभिव्यक्ति प्रायः भावात्मक स्तर पर थी, भले ही वह भावात्मकता कभी रागमय थी तो कभी विरागमय- कभी आह्लाद पूर्ण थी तो कभी करूणा विगलित। सामान्यतः इसी मूल भावना को लेकर साहित्य में बहुविध गीतों और गीतात्मक रचनाओं का विकास हुआ।

आधुनिक युग में इस गीत शब्द ने शाब्दिक तथा आर्थिक दोनों ही प्रकार से अनेक रूप धारण किया है- "गीत" शब्द का न केवल अर्थ और प्रभाव की दृष्टि से विकास हुआ है वरन् रूपात्मक दृष्टि से भी वह उतना ही विकसित हुआ है गीत से गीति-गीत काव्य अथवा गीति काव्य, प्रगीति अथवा प्रगीति काव्य, प्रगीत मुक्तक जैसे शब्दों का निर्माण हुआ है। ये शब्द अपनी अर्थवत्ता में 'गीत' की मूल भावना को छिपाए हुए भी अर्थ की दृष्टि से दिशा वैविध्य को प्राप्त होते रहे हैं। सम्प्रति गीति काव्य अथवा प्रगीति काव्य जैसे शब्दों का प्रयोग अधिकाँशतः होने लगा है। जिसका सम्बन्ध भी अन्य भाषाओं की समकक्षता में देखा जाने लगा है। कुछ लोगों ने गीत और प्रगीत जैसे शब्दों में विभेद प्रकट करने की चेष्टा की है। किन्तु व्यावहारिक रूप में इन शब्दों का प्रयोग करते समय वे स्वतः न तो सजग हैं और न ही स्पष्ट भी। इसलिए गीत काव्य में स्वरूप और उसके विकास को स्पष्ट करने के लिए गीत की मूल भावना को समझना आवश्यक है। 'गीति काव्य' के विकसित अर्थ पर दृष्टिपात करने से यह ज्ञात होता है कि 'ग्रीस' में ईसा से पाँचवी शती पूर्व गेय रचनाओं के लिए (Lyric) शब्द का प्रयोग किया जाता था। यह गेय रचना 'लायर' नामक वाद्य यन्त्र के सहारे गाई जाती थी।"4

आरम्भिक गेय कविताएँ राजनीति परक थी। ..... "लिरिक कविता ने ग्रीस में बराबर अपना गेय रूप कायम रखा। उसकी भाषा सरल और सुगम थी- आम लोगों की। उसका छन्द और उसकी शब्द रचना सभी सहज थे। उसमें साधारण जनों के हर्ष, विषाद, संयोग, वियोग, प्रणय, क्रोध आदि वर्णित होते थे। सर्वथा निजी रूप से गेयता उसका आवश्यक गुण था।"5

"गीतिकाव्य" शब्द साधारणतया तो उस समूची काव्य रचना के लिए व्यवह्तत होता था जो वाद्य यन्त्रों (Lyre) के सहारे गाई जाती थीं- फिर भी हीगल आदि अधिकाँश पाश्चात्य चिन्तक उसे एक ऐसी व्यक्ति सापेक्ष्य रचना के रूप में स्वीकार करते रहे जिसमें भावना के सहसा उद्रेक के साथ तीर की -- स्वानुभूति की गहराई, हृदय संवेगों का सहज स्वाभाविक उच्छलन एवं संगीत चेतना समाहित थी।"6 
हिन्दी में गीति काव्य 'लिरिक' के तत्त्व बोध के लिए निर्मित आधुनिक शब्द है जिसका मूलभूत आधार गीत अथवा गीतकाव्य है। गीत का प्रयोग प्राचीनतम है और नाट्यशास्त्र में इसके प्रयोग मिलते हैं। ........ गीतिकाव्य पश्चिम से आया हुआ विधान है जिसकी वहाँ संज्ञा थी (Lyric)। 'लिरिक' के अर्थ विकास का इतिहास गीतिकाव्य के तात्विक विवेचन के लिए आवश्यक होगा।"7 "काव्य अपनी आरम्भिक अवस्था में गीत ही था। आरम्भ से लेकर आज तक इस गीत अथवा गीति काव्य की महत्ता उसके संक्षेपीकरण में रखी है। सामान्यतः कवि की वैयक्तिक भाव धारा और अनुभूति को उसके अनुरूप लयात्मक अभिव्यक्ति देने के विधान को गीति काव्य कहते हैं। वह उन पूर्ण और समग्र क्षणें की वाणी है जिनकी स्थिति में वे क्षण ही पूर्ण और समग्र जीवन प्रतीत होते हैं ....... कला की कृत्रिमता भी इतनी सहज और नैसर्गिक रहती है कि उसमें सहजता का ही बोध संभव होता है।"8

गीतिकाव्य को रचना के व्यापक परिवेश में देखना ही उपयुक्त होगा। केवल गेयता से युक्त गीतों को गीतिकाव्य की संज्ञा नहीं दी जा सकती बल्कि इस प्रकार के गीतों को गीति काव्य के एक भेद के रूप में ही देखा जा सकता है।

जहाँ तक गीतिकाव्य की वैयक्तिक आत्माभिव्यक्ति का प्रश्न है निश्चय ही इस वैयक्तिकता के मानदण्ड भी बदल गए हैं यह वैयक्तिकता कवि के आत्मपरिवेश के विस्तार के साथ ही साथ विस्तृत होती गई और उसमें समाज का एक बहुत बड़ा वर्ग समहित होता गया है। इसीलिए वैयक्तिकता और आत्मपरकता के घेरे में निराला की अधिसंख्य रचनाओं से लेकर आज तक के प्रगतिवादी जनवादी काव्य को अलग नहीं किया जा सकता।

निराला ने इस रचना विधा में कवित्व और संगीत की समरूप योजना पर बल देते हुए कहा "प्राचीन गवैयों की शब्दावली, संगीत की संगति की रक्षा के लिए, किसी तरह जोड़ दी जाती थी, इसीलिए उसमें काव्य का एकान्त अभाव रहता था। आज तक उनका यह दोष प्रदर्शित होता है। मैंने अपनी शब्दावली को काव्य के स्वर में भी मुखर करने की कोशिश की है। हृस्व-दीर्घ की घट-बढ़ के कारण पूर्ववर्ती गवैये शब्दाकारों पर जो लांछन लगता है, उससे भी बचने का प्रयत्न किया है। दो-एक स्थलों को छोड़कर अन्यत्र सभी जगह संगीत के छन्दः शास्त्र की अनुवर्तिता की है। भाव प्राचीन होने पर भी प्रकाशन का नवीन ढंग लिए हुए हैं। जो संगीत कोमल, मधुर और उच्च भाव तद्नुकूल भाषा और प्रकाशन से व्यक्त होता है, उसके साफल्य की मैंने कोशिश की है।"9 
निराला के द्वारा प्रस्तुत गीति काव्यांगों में से मौलिकता और भावानुकूल भाषा तो काव्य-मात्र के लिए वांछित हैं, किन्तु काव्य-शोभा की योजना के लिए संगीत का उपयुक्त आश्रय लेना उसकी अपनी विशेषता है।

गीतिकवियों में महादेवी वर्मा का स्थान 'निराला' के पश्चात् ही आता है। पंत जी ने 'गीत-गद्य' के नाम से एक नवीन काव्य विधा का उद्भावन किया। महादेवी वर्मा ने काव्य रचना के रूपों मे से गीतिकाव्य के स्वरूप का समृद्ध विवेचन किया।" सुख-दुख की भावावेशमयी अवस्था- विशेष का गिने चुने शब्दों में स्वर-साधना के उपयुक्त चित्रण कर देना ही गीत है।"

"गेयता में ज्ञान का क्या स्थान है यह भी प्रश्न है। बुद्धि के तर्क-क्रम से जिस ज्ञान की की उपलब्धि हो सकती है उसका भार गीत नहीं संभाल सकता, पर तर्क से परे इन्द्रियों की सहायता के बिना भी हमारी आत्मा अनायास ही जिस सत्य का ज्ञान प्राप्त कर लेती है उसकी अभिव्यक्ति में गेय स्वर-सामंजस्य का विशेष महत्त्व रहा है।"10

गीतिकाव्य में तीन गुणों की स्थिति होनी आवश्यक है- (1) आत्माभिव्यक्ति अर्थात् वैयक्तिक सुख-दुख का कथन (2) बौद्धिक तर्क क्रम के स्थान पर अनुभूति से प्राप्त ज्ञान (3) गेयता अथवा स्वरों को आरोह-अवरोह क्रम।

गीतिकाव्य के अन्य प्रकारों में महादेवी वर्मा ने लोक गीत के स्वरूप की भी व्याख्या की है। उन्होंने रहस्यगीत (सुगणोन्मय गीत तथा निर्गुण गीत) के साथ-साथ राष्ट्रगीत का भी उल्लेख किया है। रहस्यगीत की प्रवृत्तियों की व्याख्या करते हुए वह कहती हैं "रहस्यगीतों का मूलाधार भी आत्मानुभूत अखण्ड चेतन है तथा रहस्य गीतों में आनन्द की अभिव्यक्ति के सहारे ही हम चित् और सत् तक पहुँचते हैं।"11

इसी परम्परा में रामकुमार वर्मा ने भी गीतिकाव्य के स्वरूप की समीक्षा की है। "प्रसाद जी का आँसू शीर्षक लेख में प्रगीतात्मक रचना की विशेषताओं के विषय में कहते हैं" आँसू एक उच्च कोटि का गीतिकाव्य है इसमें भावना की एकरूपता, अनुभूति की तीव्रता तथा मधुरसंगीत आदिगीतिकाव्य के अनेक गुण पाए जाते हैं।"12

गीत की सफल रचना के लिए कवि को इन तीनों गुणों पर समान रूप से ध्यान देना होता है और इनमें से अनुभूति प्रबलता को गीत का मूल हेतु माना जा सकता है। संगीत की मधुर स्वर-लहरी के माध्यम से भावना की अभिव्यक्त अनुभूति का स्वाभाविक फल है।

प्रगीतकार 'बच्चन' ने गीत के स्वरूप को इन शब्दों में प्रकट किया "प्रत्येक गीत को सर्व स्वतंत्र अपराश्रित और अपने में ही परिपूर्ण मान कर पढ़ा या गाया जाता है और उसका रस लिया 
जाता है। अब यह गीतकार का काम है कि गीतों की परिमित परिधि के भीतर ही भावों का उद्रेक और विकास कर उन्हें वांछित परिणति तक पहुँचा दें।"13 इस उक्ति में गीतिकाव्य के दो गुणों को स्पष्ट किया गया पहला उसमें किसी एक ही भाव को स्वतन्त्र और परिपूर्ण अभिव्यक्ति प्रदान की जाती है, और दूसरे उसके अध्ययन से भावक को रस अथवा आनन्द की उपलब्धि होती है। वस्तुतः ये दोनों गुण नवीन नहीं है वरन् कवियों की परम्परा में इनमें से पहले गुण का निर्दोष सर्वप्रथम हरिवंश राय बच्चन ने ही किया है। 'बच्चन' की प्रतिभा मूलतः गीतात्मक है। गीतिकाव्य के लेखकों में जो सुबोधता उनकी रचनाओं में पाई जाती है वह अन्यत्र दुर्लभ है। प्रतीक विधान उनकी रचनाओं में हिन्दी के बहुत से कवियों की अपेक्षा अधिक सरस, सहज और सफल है। हिन्दी के छन्दों के अतिरिक्त उर्दू के बहुत से गीतों में ध्वनि का सौन्दर्य तो नहीं पर एक प्रकार की गूँज की मधुरता है, जो अंतःकरण में देर तक मंडराती रहती है।

उत्तर छायावाद काल के कवियों में नरेन्द्र शर्मा की ख्याति प्रवासी के गीत के प्रकाशन के साथ आरंभ होती है इस कृति ने एक सफल गीतकार के रूप में उन्हें सदैव के लिए प्रतिष्ठित कर दिया।

आलोच्य कवियों ने गीतिकाव्य के स्वरूप की विविध व्याख्या तो की परन्तु उनकी धारणाओं का विश्लेषण करने पर भी गीत की परिभाषा पूर्ण नहीं हो सकती है क्योंकि गीत केवल वह रचना नहीं है जिसमें भाव-विशेष की स्वतन्त्र परिपूर्ण, लयबद्ध तथा आनन्दात्मक अभिव्यक्ति हो उसमें आत्माभिव्यक्ति आदि अन्य तत्वों का भी महत्त्वपूर्ण योग रहता है।

\section{सन्दर्भ}



